\title{
Usefulness of ECG to differentiate apical hypertrophic cardiomyopathy from non-ST elevation acute coronary syndrome
}

\author{
Yirao $\mathrm{Tao}^{1 \dagger}$, Jing $\mathrm{Xu}^{2 \dagger}$, Samira Yerima Bako ${ }^{1}$, Xiaobo $\mathrm{Yao}^{2}$ and Donghui Yang ${ }^{*^{*}}$ (D)
}

\begin{abstract}
Background: Apical hypertrophic cardiomyopathy (ApHCM) is a phenotypic variant of nonobstructive HCM. ApHCM is characterized by left ventricular hypertrophy involve the distal apex. The electrocardiographic character of ApHCM can mimic non-ST elevation acute coronary syndrome (NSTEACS), triggering a series of studies and treatments that may be unnecessary. This study aimed to clarify the electrocardiogram (ECG) differences between the two diseases.
\end{abstract}

Methods: Initial ECG recordings of 41 patients with ApHCM and 72 patients with NSTEACS were analyzed retrospectively. We analyzed the voltage of negative $T$ (neg $T$ ) and R wave, the change of ST-segment as well as the number of leads with neg T wave in the 12-lead ECGs.

Results: Across the 12-lead ECGs, the magnitude of R wave significantly differed between ApHCM and NSTEACS in 10 leads excluding leads aVR and V1. ApHCM was associated with a greater maximal amplitude of R wave in lead V5 ( $3.13 \pm 1.08$ vs. $1.38 \pm 0.73 \mathrm{mV}, P<0.001)$. The magnitude of T wave significantly differed between ApHCM and NSTEACS in 10 leads excluding leads $\|$ and V1. ApHCM was associated with a greater maximal amplitude of neg T wave in lead V4 ( $0.85 \pm 0.69$ vs. $0.35 \pm 0.23 \mathrm{mV}, P<0.001)$. The frequency of giant neg $\mathrm{T}(1 \mathrm{mv}$ or more) wave was higher in ApHCM (36.5\% vs. $0 \%, P<0.001)$. The magnitude of ST-segment deviation significantly differed between ApHCM and NSTEACS in 10 leads excluding leads aVF and V2. ApHCM was associated with a greater maximal amplitude of STsegment depression in lead V5 $(0.19 \pm 0.07$ vs. $0.03 \pm 0.06 \mathrm{mV}, P<0.001)$. The number of leads with neg $T$ wave also differed between ApHCM and NSTEACS (6.75 \pm 1.42 vs. $6.08 \pm 1.51, P=0.046)$. The sum of $R$ wave in lead V5, neg T wave in lead V6 and ST-segment depression in lead V4 > $2.585 \mathrm{mV}$ identified ApHCM with 90.2\% sensibility and 87.5\% specificity, representing the highest diagnostic accuracy.

Conclusions: Compared with NSTEACS patients, ApHCM patients presented higher $\mathrm{R}$ and neg $\mathrm{T}$ wave voltage as well as a greater ST-segment depression in the 12-lead ECG. The ECG characteristics can help to differentiate ApHCM from NSTEACS in clinical setting.

Keywords: Apical hypertrophic cardiomyopathy, Non-ST elevation acute coronary syndrome, Electrocardiogram

\footnotetext{
* Correspondence: yangdonghui1@126.com

${ }^{\dagger}$ Yirao Tao and Jing Xu are co-first authors

'Department of Cardiology, The Second Affiliated Hospital of Dalian Medical University, Dalian, Liaoning, China

Full list of author information is available at the end of the article
}

(c) The Author(s). 2020 Open Access This article is licensed under a Creative Commons Attribution 4.0 International License, which permits use, sharing, adaptation, distribution and reproduction in any medium or format, as long as you give appropriate credit to the original author(s) and the source, provide a link to the Creative Commons licence, and indicate if changes were made. The images or other third party material in this article are included in the article's Creative Commons licence, unless indicated otherwise in a credit line to the material. If material is not included in the article's Creative Commons licence and your intended use is not permitted by statutory regulation or exceeds the permitted use, you will need to obtain permission directly from the copyright holder. To view a copy of this licence, visit http://creativecommons.org/licenses/by/4.0/ The Creative Commons Public Domain Dedication waiver (http://creativecommons.org/publicdomain/zero/1.0/) applies to the data made available in this article, unless otherwise stated in a credit line to the data. 


\section{Background}

Apical hypertrophic cardiomyopathy (ApHCM) is complex phenotypic variant of the classical hypertrophic cardiomyopathy [1]. It can be asymptomatic or present with dyspnea, chest pain, syncope as well as severe diastolic dysfunction even sudden cardiac death [2-4]. Given its diverse presentation forms, clinical evaluation alone cannot be relied upon. Understanding the unique electrocardiogram (ECG) features of ApHCM can be of assistance in the diagnostic process of this uncommon disease.

Because of similar clinical manifestations and large negative $\mathrm{T}$ (neg $\mathrm{T}$ ) waves in precordial leads on ECG, most previous case reports misdiagnosed ApHCM as non-ST elevation acute coronary syndrome (NSTEACS) [5-11]. Differentiation of these two diseases can be challenging, but has an important role in the selection of an appropriate treatment strategy. The 12-lead ECG is the simplest and widely used clinical diagnostic test. Several ECG features of ApHCM have been reported which may help to make these distinctions $[12,13]$. These include higher $\mathrm{T}$ wave voltage and peak voltage, $\mathrm{T}$ wave asymmetry and higher R waves. Nevertheless, previous studies were restricted to case reports or a small group of patients, the serial ECG differences between ApHCM and NSTEACS have not been sufficiently elucidated.

Our aim was to explore the ECG patterns in ApHCM patients and compare them with NSTEACS patients to distinguish between the two diagnoses, thereby helping choose a more appropriate treatment strategy and finally improving clinical outcome.

\section{Methods \\ Participants}

We retrospectively studied 113 consecutive patients (41 patients with ApHCM and 72 patients with NSTEACS) who were admitted to our Cardiology department within 7 days from symptom onset between April 2015 and April 2019. Exclusion criteria included ventricular pacing, atrial fibrillation or flutter and left or right bundle branch block. The basic data of gender, age, history of smoking and alcohol, diabetes and hypertension were also recorded. This study was approved by our Institutional Ethics Committee and all participants provided informed consent according to the Declaration of Helsinki.

\section{ApHCM group}

Definition of ApHCM relies on demonstrating left ventricular hypertrophy predominating in the distal apex by cardiovascular magnetic resonance imaging or transthoracic echocardiography, with a wall thickness $\geq 15 \mathrm{~mm}$ of the apex and maximal apical/posterior wall ratio $\geq 1.5[14,15]$.

\section{NSTEACS group}

NSTEACS group included subjects with unstable angina (UA) and acute non-ST segment elevation myocardial infarction (NSTEMI) [16]. All patients presented with precordial $\mathrm{T}$ waves inversion on admission ECG and have an ischemic symptom, such as new-onset, rest, or increasing angina. Patients with left ventricular hypertrophy diagnosed by cardiovascular magnetic resonance or transthoracic echocardiography were excluded from this group.

\section{ECG evaluation}

A standard 12-lead ECG on admission was recorded at a $10 \mathrm{~mm} / \mathrm{mV}$ amplitude and a $25 \mathrm{~mm} / \mathrm{s}$ speed. QT interval was corrected using the Bazett formula [17]. The STsegment deviation was measured manually $0.08 \mathrm{~s}$ after the J-point in each lead [18]. We analyzed the following ECG differences: (1) R wave amplitude in 12 leads; (2) T wave amplitude in 12 leads; (3) amplitude of STsegment deviation in 12 leads; (4) giant neg $\mathrm{T}$ wave (1 $\mathrm{mV}$ or morein any ECG lead [19]); (5) the number of leads with neg $\mathrm{T}$ wave; (6) total amplitude of neg $\mathrm{T}$ waves. All ECGs were measured by a single investigator who was blinded to clinical information. The average values came from three continuous sinus beat.

\section{Statistics}

Continuous data were described as mean $( \pm \mathrm{SD})$ and compared by Mann-Whitney U-test or Student's T-test. Categorical data are described as numbers and percentages and compared by Fisher's exact test or Chi-square test. Youden's index which derived from receiver operator characteristic (ROC) curves evaluated the cut-off value, while the area under curve (AUC) evaluated which ECG marker represented the highest diagnostic accuracy. $P$-value $<0.05$ was considered significant. SPSS, version 22.0 software was used to manage the data.

\section{Results \\ Study group}

The baseline characteristics were presented in Table 1 . The mean $( \pm$ SD) age was $69.55( \pm 10.75)$ years. $46.9 \%$ of participants were men. Patients with ApHCM were more likely to be male and had a larger left atrium, higher left ventricular ejection fraction (LVEF), thicker left ventricular posterior wall (LVPW) and interventricular septal (IVS) as well as a lower prevalence of diabetes mellitus than those in NSTEACS group. Other characteristics did not differ between ApHCM and NSTEACS.

\section{ECG findings}

QRS interval and QTc interval did not differ significantly between ApHCM and NSTEACS. The magnitude of $\mathrm{R}$ wave significantly differed between ApHCM 
Table 1 Baseline Characteristics

\begin{tabular}{llll}
\hline & ApHCM $(n=41)$ & NSTEACS $(n=72)$ & $P$-value \\
\hline Men & $27(65.9)$ & $26(36.1)$ & 0.002 \\
Age (years) & $68.29 \pm 11.12$ & $70.27 \pm 10.55$ & 0.384 \\
Smoking & $12(29.3)$ & $22(30.6)$ & 0.886 \\
Drinking & $5(12.2)$ & $7(9.7)$ & 0.682 \\
Hypertension & $28(68.3)$ & $54(75)$ & 0.442 \\
Diabetes mellitus & $5(12.2)$ & $21(29.2)$ & 0.039 \\
LAD $(\mathrm{mm})$ & $42.09 \pm 4.54$ & $39.38 \pm 4.71$ & 0.004 \\
LVEDd $(\mathrm{mm})$ & $47.82 \pm 3.93$ & $47.40 \pm 5.58$ & 0.320 \\
LVEDs $(\mathrm{mm})$ & $29.97 \pm 3.01$ & $32.08 \pm 5.85$ & 0.158 \\
IVS $(\mathrm{mm})$ & $11.65 \pm 2.42$ & $9.61 \pm 1.21$ & $<0.001$ \\
LVPW $(\mathrm{mm})$ & $9.82 \pm 1.04$ & $9.25 \pm 0.91$ & 0.001 \\
LVEF $(\%)$ & $65.65 \pm 4.89$ & $59.13 \pm 9.32$ & $<0.001$ \\
E/A & $0.97 \pm 0.59$ & $0.84 \pm 0.37$ & 0.853 \\
E/Em & $10.12 \pm 5.92$ & $10.10 \pm 4.97$ & 0.795 \\
\hline AbbreVlation & ALa &
\end{tabular}

Abbreviation: $A$ Late diastolic inflow velocity, ApHCM Apical hypertrophic cardiomyopathy, E Early diastolic inflow velocity, Em Early diastolic annular tissue velocity, IVS Interventricular septal, LAD Left atrial diameter, LVEDd Left ventricular end diastolic diameter, LVEDs Left ventricular end systolic diameter, LVEF LEFT ventricular ejection fraction, LVPW Left ventricular posterior wall, NSTEACS Non-ST elevation acute coronary syndrome

and NSTEACS in 10 leads, excluding leads aVR and V1. ApHCM was associated with a greater maximal amplitude of $\mathrm{R}$ wave $(3.13 \pm 1.08$ vs. $1.38 \pm 0.73 \mathrm{mV}$, $P<0.001)$ in lead V5. The comparison of QRS interval, QTc interval and $\mathrm{R}$ wave were shown in Table 2 and Fig. 1. Neg T waves were consistently observed in leads $\mathrm{I}$, aVL and V2-V6 in ApHCM. The magnitude of T wave significantly differed between ApHCM and

Table 2 The comparison of QRS interval, QTc interval and R wave between ApHCM and NSTEACS in ECG

\begin{tabular}{llll}
\hline & ApHCM $(n=41)$ & NSTEACS $(n=72)$ & $P$-value \\
\hline QRS interval (ms) & $97.26 \pm 11.20$ & $95.69 \pm 14.54$ & 0.174 \\
QTC interval (ms) & $441.24 \pm 26.34$ & $438.75 \pm 43.53$ & 0.254 \\
R wave in I (mV) & $1.31 \pm 0.42$ & $0.83 \pm 0.39$ & $<0.001$ \\
R wave in II (mV) & $1.55 \pm 1.42$ & $0.66 \pm 0.40$ & $<0.001$ \\
R wave in III (mV) & $0.48 \pm 0.43$ & $0.26 \pm 0.23$ & 0.004 \\
R wave in aVR (mV) & $0.11 \pm 0.12$ & $0.10 \pm 0.12$ & 0.299 \\
R wave in aVL (mV) & $0.73 \pm 0.45$ & $0.60 \pm 0.36$ & 0.121 \\
R wave in aVF (mV) & $0.72 \pm 0.47$ & $0.38 \pm 0.28$ & $<0.001$ \\
R wave in V1 (mV) & $0.33 \pm 0.35$ & $0.22 \pm 0.28$ & 0.029 \\
R wave in V2 (mV) & $1.51 \pm 0.86$ & $0.71 \pm 0.70$ & $<0.001$ \\
R wave in V3 (mV) & $2.38 \pm 1.04$ & $1.03 \pm 0.73$ & $<0.001$ \\
R wave in V4 (mV) & $3.10 \pm 1.13$ & $1.33 \pm 0.76$ & $<0.001$ \\
R wave in V5 (mV) & $3.13 \pm 1.08$ & $1.38 \pm 0.73$ & $<0.001$ \\
R wave in V6 (mV) & $2.45 \pm 0.95$ & $1.20 \pm 0.66$ & $<0.001$ \\
\hline Abbreviation: ApHCM Apical hypertrophic cardiomyopathy, NSTEACS Non-ST
\end{tabular}

Abbreviation: ApHCM Apical hypertrophic cardiomyopathy, NSTEACS Non-ST elevation acute coronary syndrome
NSTEACS in 10 leads, excluding leads II and V1. ApHCM was associated with a greater maximal amplitude of neg $\mathrm{T}$ wave $(0.85 \pm 0.69$ vs. $0.35 \pm 0.23 \mathrm{mV}$, $P<0.001)$ in lead V4. The frequency of giant neg $\mathrm{T}$ wave was higher in ApHCM $(36.5 \%$ vs. $0 \%, P<0.001)$. Besides, a greater total magnitude of neg $\mathrm{T}$ waves (3.38 \pm 1.75 vs. $1.47 \pm 0.85 \mathrm{mV}, P<0.001)$ and a larger number of leads with neg $\mathrm{T}$ wave $(6.75 \pm 1.42$ vs. $6.08 \pm$ 1.51, $P=0.046)$ were found in ApHCM. The comparisons of $\mathrm{T}$ wave were shown in Table 3 and Fig. 2. STsegments elevation in leads aVR, V1 and ST-segments depression in leads I, V4-V6 were consistently observed in ApHCM. The magnitude of ST-segment deviation significantly differed between ApHCM and NSTEACS in 10 leads, excluding leads aVF and V2. ApHCM was associated with a greater maximal amplitude of STsegment depression $(0.19 \pm 0.07$ vs. $0.03 \pm 0.06 \mathrm{mV}$, $P<0.001)$ in lead V5. The comparisons of ST-segment deviation in 12 leads were shown in Table 4 and Fig. 3.

$\mathrm{R}$ wave in lead V5 represent the highest sensitivity (90.2\%) for ApHCM compared with the other leads with the cut-off value of $2.07 \mathrm{mV}$.Neg T wave in lead V6 had the highest sensitivity (95.1\%) for ApHCM and the cutoff value was $0.185 \mathrm{mV}$. Considering ST-segment depression, the highest sensitivity (83.3\%) for ApHCM was at lead V4 with the cut-off value of $0.05 \mathrm{mV}$. The summation of $\mathrm{R}$ wave in lead V5, neg $\mathrm{T}$ wave in lead V6 and ST-segment depression in lead V4 $>2.585 \mathrm{mV}$ identified ApHCM with $90.2 \%$ sensitivity and $87.5 \%$ specificity, which was showed in Table 5. Representative ECGs of each group were shown in Fig. 4.

\section{Discussion}

The current study revealed that the peak voltage of neg $\mathrm{T}$ and giant $\mathrm{R}$ clearly differed between ApHCM and NSTEACS, which occurred most frequently in leads V2V6. We also showed that degree of ST-segment depression differed, particularly in leads V3-V6, between the two diseases. To our best knowledge, this is the first study to examine the change of ST-segment in patients with ApHCM and NSTEACS.

ApHCM has been recognized as Japanese-variant of hypertrophic cardiomyopathy since its high prevalence in the Japanese population $[20,21]$. The etiology of ApHCM is multifactorial, with studies suggesting variants with a genetic predisposition and exclusive development during adulthood [22, 23]. ApHCM presents some particular electrocardiographic findings including deep inversion of the $\mathrm{T}$ waves (giant $\mathrm{T}$ waves) and the increase of the QRS complex voltage in the precordial leads [13]. However, in clinical setting, the ApHCM patients with giant neg $\mathrm{T}$ waves recorded in the ECG were always suspected of having acute coronary syndrome (ACS) [24]. Rogers reported a 61-year-old man admitted 


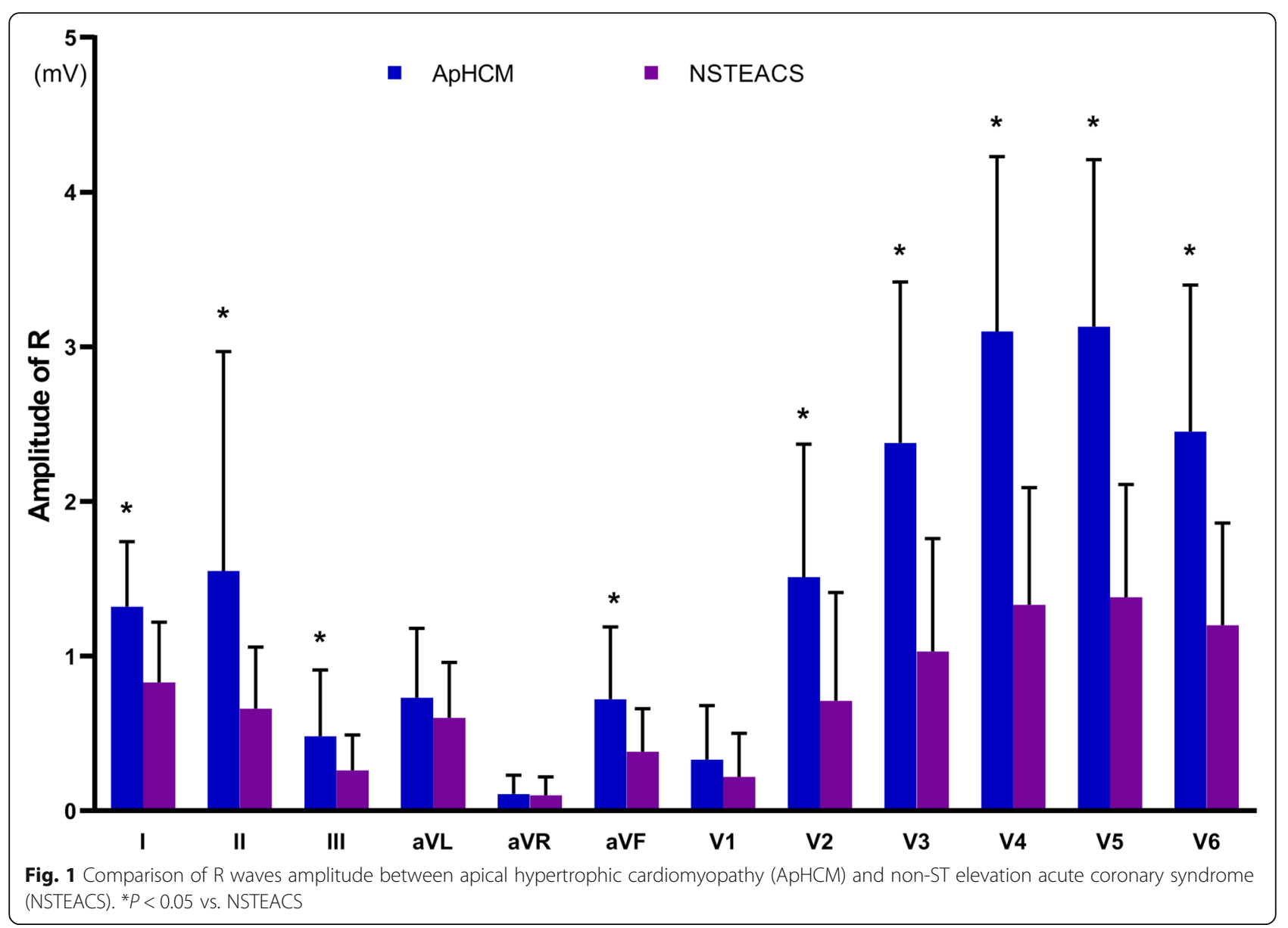

Table 3 The comparison of T wave between ApHCM and NSTEACS in ECG

\begin{tabular}{llll}
\hline & ApHCM $(n=41)$ & NSTEACS $(n=72)$ & $P$-value \\
\hline T wave in I (mV) & $-0.14 \pm 0.09$ & $-0.02 \pm 0.10$ & $<0.001$ \\
T wave in II (mV) & $-0.03 \pm 0.11$ & $0.01 \pm 0.09$ & 0.070 \\
T wave in III (mV) & $0.13 \pm 0.13$ & $0.03 \pm 0.12$ & $<.001$ \\
T wave in aVR (mV) & $0.08 \pm 0.08$ & $0.01 \pm 0.07$ & $<.001$ \\
T wave in aVL (mV) & $-0.12 \pm 0.12$ & $-0.02 \pm 0.10$ & 0.001 \\
T wave in VVF (mV) & $0.08 \pm 0.17$ & $0.02 \pm 0.09$ & 0.002 \\
T wave in V1 (mV) & $0.02 \pm 0.10$ & $0.01 \pm 0.11$ & $<.230$ \\
T wave in V2 (mV) & $-0.31 \pm 0.30$ & $-0.04 \pm 0.23$ & $<0.001$ \\
T wave in V3 (mV) & $-0.66 \pm 0.43$ & $-0.32 \pm 0.25$ & $<0.001$ \\
T wave in V4 (mV) & $-0.85 \pm 0.69$ & $-0.35 \pm 0.23$ & $<.001$ \\
T wave in V5 (mV) & $-0.67 \pm 0.37$ & $-0.28 \pm 0.18$ & $<0.001$ \\
T wave in V6 (mV) & $-0.48 \pm 0.38$ & $-0.18 \pm 0.13$ & $<.001$ \\
Giant neg T wave (number of cases/\%) & $15(36.5)$ & 0 & $<0.001$ \\
Number of leads with neg T wave & $6.75 \pm 1.42$ & $6.08 \pm 1.51$ & 0.046 \\
Total amplitude of neg T waves (mV) & $3.38 \pm 1.75$ & $1.47 \pm 0.85$ & $<0.001$ \\
\hline
\end{tabular}

Abbreviation: ApHCM Apical hypertrophic cardiomyopathy, NSTEACS Non-ST elevation acute coronary syndrome, neg Negative 


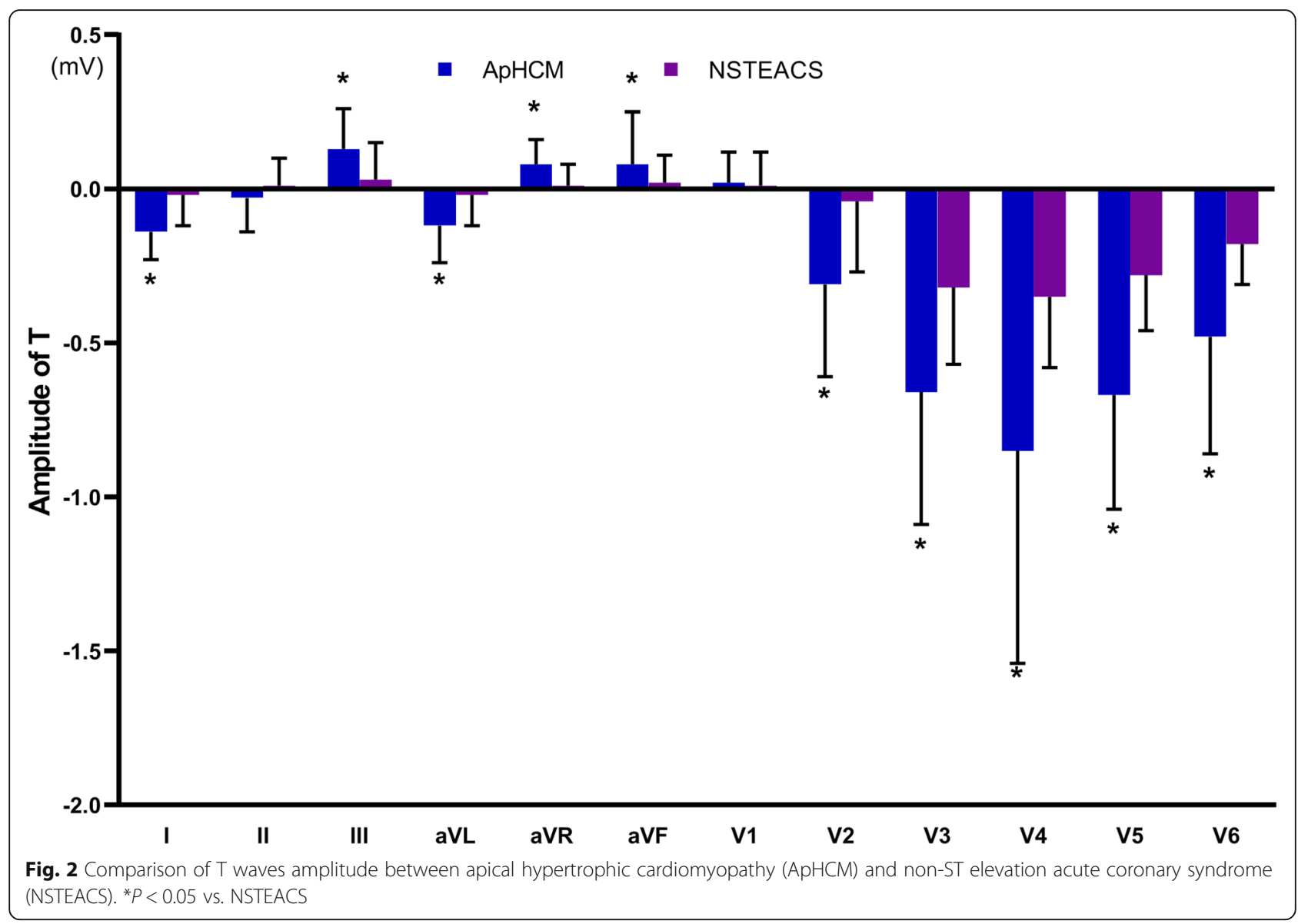

Table 4 The comparison of ST-segment deviation in 12 leads between ApHCM and NSTEACS in ECG

\begin{tabular}{llll}
\hline & ApHCM $(n=41)$ & NSTEACS $(n=72)$ & $P$-value \\
\hline ST-segment in I (mV) & $-0.10 \pm 0.03$ & $-0.01 \pm 0.03$ & $<0.001$ \\
ST-segment in II (mV) & $-0.04 \pm 0.05$ & $-0.01 \pm 0.03$ & 0.002 \\
ST-segment in III (mV) & $0.06 \pm 0.06$ & $0.00 \pm 0.02$ & $<0.001$ \\
ST-segment in aVR (mV) & $0.07 \pm 0.04$ & $0.01 \pm 0.03$ & $<0.001$ \\
ST-segment in aVL (mV) & $-0.04 \pm 0.07$ & $-0.01 \pm 0.02$ & $<0.001$ \\
ST-segment in aVF (mV) & $0.01 \pm 0.06$ & $-0.01 \pm 0.04$ & 0.057 \\
ST-segment in V1 (mV) & $0.07 \pm 0.05$ & $0.01 \pm 0.04$ & $<0.001$ \\
ST-segment in V2 (mV) & $0.02 \pm 0.09$ & $0.01 \pm 0.04$ & 0.054 \\
ST-segment in V3 (mV) & $-0.08 \pm 0.10$ & $0.00 \pm 0.05$ & $<0.001$ \\
ST-segment in V4 (mV) & $-0.15 \pm 0.01$ & $-0.02 \pm 0.05$ & $<0.001$ \\
ST-segment in V5 (mV) & $-0.19 \pm 0.07$ & $-0.03 \pm 0.06$ & $<0.001$ \\
ST-segment in V6 (mV) & $-0.18 \pm 0.05$ & $-0.04 \pm 0.07$ & $<0.001$ \\
\hline Abbreviation: ApHCM Apical hypertrophic cardiomyopathy, NSTEACS Non-ST
\end{tabular}

Abbreviation: ApHCM Apical hypertrophic cardiomyopathy, NSTEACS Non-ST elevation acute coronary syndrome with chest pain, his ECG showed a biphasic $\mathrm{T}$ wave in lead V2, neg $\mathrm{T}$ waves in leads II and aVL, deep symmetrical T-wave inversions and ST depressions in leads V3V6 [11]. He was suspected of ACS undergoing an emergent cardiac catheterization which revealed no coronary artery disease but a "spade like" pattern suggestive of ApHCM. Meghrajani reported a 66-year-old woman whose initial ECG showed $\mathrm{T}$ wave inversions in the lateral leads was diagnosed with type 2 myocardial infarction [25]. Coronary angiogram as well as cardiac left ventriculogram showed apical hypertrophy without coronary artery occlusion. From the ECG point of view, especially inverted $\mathrm{T}$ waves in V3-V6, ApHCM is often difficult to differentiate from NSTEACS. Previous studies had been confined to case reports or a relatively small number of patients. Herein we conducted an observational and retrospective study, the ECG findings could be conducive to differentiate ApHCM and NSTEACS early thus preclude the need for urgent coronary angiography and make accurate diagnosis and treatment essential for improved outcome.

To our knowledge, only the study reported by CHILLIK scrutinized ECG differences between ApHCM and NSTEMI [12]. They compared ECG changes between 19 


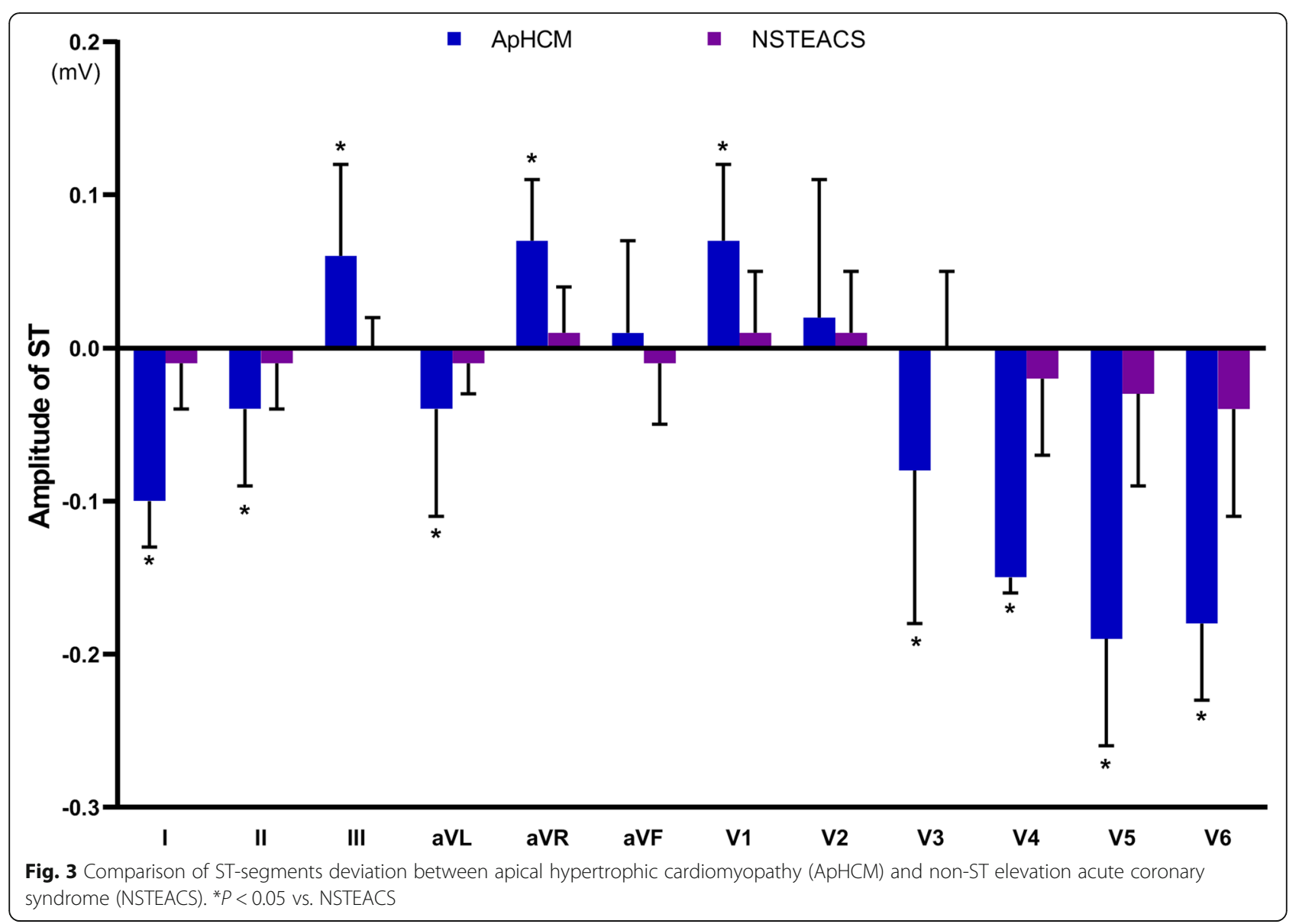

patients with ApHCM and 19 patients with NSTEMI. They assessed neg $\mathrm{T}$ waves in leads V1-V6 showing a greater $\mathrm{T}$-wave asymmetry. However, their study included only a small number of patients and they did not examine differences in ST-segment and the distributions or numbers of leads with neg $\mathrm{T}$ waves. Moreover, most previous studies showed T-wave typically displays $>10 \mathrm{~mm}$ inversions within the anterolateral leads in ApHCM, most prominent in V4 and V5 yet limb leads were received little attention. We therefore evaluated the $\mathrm{R}$ and $\mathrm{T}$ waves in all 12 leads. We identified the patients with ApHCM presented higher $\mathrm{R}$ and $\mathrm{T}$ wave voltage and peak voltage, similar to previous published studies. Besides, our study showed that
ApHCM was associated with a greater ST-segment depression compared with NSTEACS. Meanwhile, the number of leads with neg $\mathrm{T}$ wave across 12-leads was more in patients with ApHCM. Giant neg $\mathrm{T}$ wave was exclusively found in ApHCM and the sum of $\mathrm{R}$ wave in lead V5, neg $\mathrm{T}$ wave in lead V6 and depressive (dep) ST-segment in lead V4 $>2.585 \mathrm{mV}$ had the highest predictive value for ApHCM. Interestingly, we found on precordial leads of ApHCM, the amplitude of T-wave inversion displayed TV4 $>$ TV $5>$ TV3, on the other hand, the amplitude of R-wave showed RV5 $>$ RV4 $>$ RV3. A series of new discoveries in our study would further facilitate differential diagnosis between ApHCM and NSTEACS.

Table 5 Predictive values of electrocardiographic variables of the diagnosis of apical hypertrophic cardiomyopathy

\begin{tabular}{|c|c|c|c|c|c|c|}
\hline & Cut off (mv) & Sensitivity & Specificity & PPV & NPV & Predictive accuracy \\
\hline R wave in lead V5 & 2.07 & $90.2 \%$ & $80.6 \%$ & $72.5 \%$ & $93.5 \%$ & $92.3 \%$ \\
\hline Neg T wave in lead V6 & 0.185 & $95.1 \%$ & $61.1 \%$ & $58.2 \%$ & $95.7 \%$ & $85.2 \%$ \\
\hline Dep ST-segment in lead V4 & 0.05 & $83.3 \%$ & $92.7 \%$ & $76.5 \%$ & $96.7 \%$ & $82.0 \%$ \\
\hline$R$ wave in lead $V 5+$ neg $T$ wave in lead $V 6+$ dep ST-segment in lead $V 4$ & 2.585 & $90.2 \%$ & $87.5 \%$ & $80.4 \%$ & $94.0 \%$ & $94.1 \%$ \\
\hline
\end{tabular}

Abbreviation: dep Depressive, neg Negative 


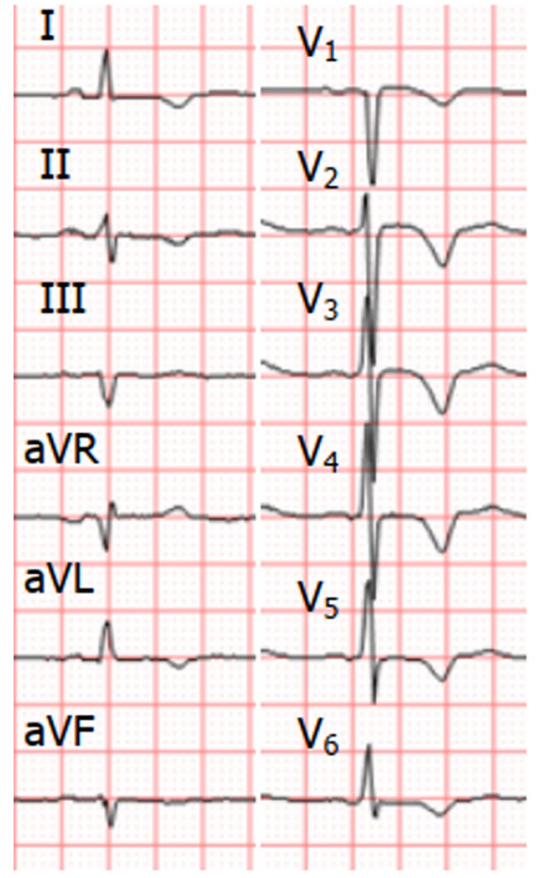

NSTEACS

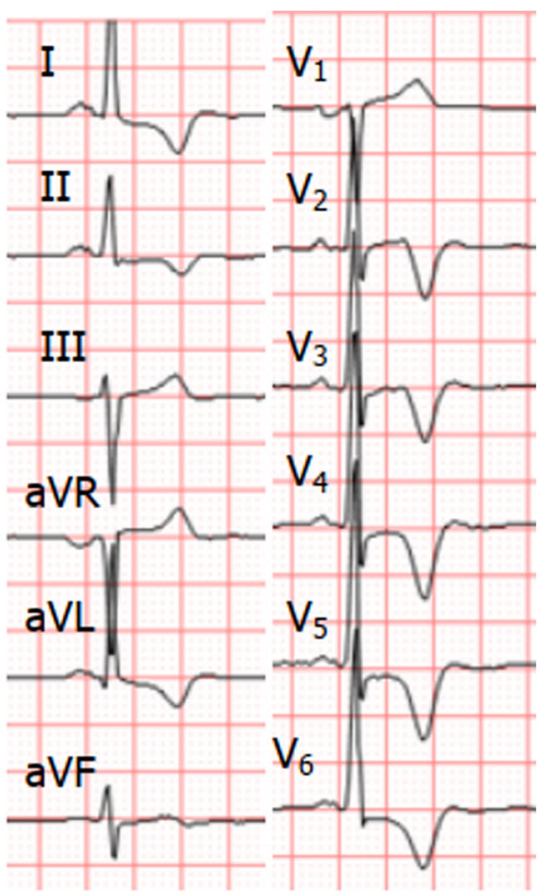

ApHCM

Fig. 4 Representative ECGs of apical hypertrophic cardiomyopathy (ApHCM) and non-ST elevation acute coronary syndrome (NSTEACS). Left (NSTEACS): negative T waves were observed in leads I, II, aVL, V1-V6. Right (ApHCM): negative T waves were observed in leads I, II, aVL, V2-V6, STsegment elevation in leads III, aVR, V1 and ST-segment depression in leads I, aVL, V4-V6

The mechanisms responsible for the ECG differences and the underlying electrophysiologic conditions between ApHCM and NSTEACS are uncertain. These voltage criteria of ApHCM may be related to both LV hypertrophy and differences in localized wall thickness leading to disparities in the duration of repolarization. ApHCM is characterized by circular LV hypertrophy, while in NSTEACS, LV hypertrophy presented at the opposite side of the myocardium because of cardiac remodeling, thus it is not a circular hypertrophy [26]. In ApHCM, the mechanism for enormous $\mathrm{R}$ waves, dramatically in V3-V4 leads, is due to the apical distribution of hypertrophy opposite to the non-muscular elements of the fibrous cardiac skeleton of the mitral valve and annular plane. This result in an unopposed depolarization vectorial depolarization forces directed towards the cardiac apex [27]. Additionally, an alternative mechanism for prominent $\mathrm{R}$ waves is increased resistivity of cardiac muscle caused by fibrosis and myofibril disarray in the hypertrophied regions. Contrary to prominent $\mathrm{R}$-waves, giant neg $\mathrm{T}$ waves isattributed to opposite vectorial orientation away from the cardiac apex. Neg T wave and dep ST-segment is considered a secondary phenomenon to high R-wave $[26,27]$.
Finally, it is important to apply these ECG rules into clinical context. Patient history is essential as ApHCM is a condition with varying clinical presentations. Active chest pain may suggest NSTEACS, whereas dyspnea usually imply ApHCM. In NSTEACS, you can find reciprocal ST changes or "mirror changes" on ECG, which is not usual in ApHCM [28]. Besides, ECG changes in $\mathrm{ApHCM}$ are generally stable against the rapid changes in ST-segment and $\mathrm{T}$ wave seen in serial ECGs of NSTEACS patients.

\section{Study limitations}

Our study was performed as a retrospective analysis and at a single center. The number of patients, particularly ApHCM patients, was relatively small. Furthermore, we have to rule out the patients falling to meet our inclusion criteria (such as sinus rhythm). Therefore, our finding may not be available for the general group of patients with ApHCM or NSTEACS. Finally, the mean age of the enrolled ApHCM patients in our study was relatively high.

\section{Conclusions}

Compared with NSTEACS, ApHCM patients presented higher voltage of $\mathrm{R}$ and neg $\mathrm{T}$ wave as well as greater 
ST-segment depression in the 12-lead ECG. Our proposed ECG characteristics can help to differentiate ApHCM from NSTEACS in clinical setting. Further studies in greater numbers of subjects are needed to verify our results.

\section{Abbreviations \\ A: Late diastolic inflow velocity; ACS: Acute coronary syndrome; ApHCM: Apical hypertrophic cardiomyopathy; AUC: Area under curve Dep: Depressive; E: Early diastolic inflow velocity; ECG: Electrocardiogram; Em: Early diastolic annular tissue velocity; IVS: Interventricular septal; LAD: Left atrial diameter; LVEDd: Left ventricular end diastolic diameter; LVEDs: Left ventricular end systolic diameter; LVEF: Left ventricular ejection fraction; LVPW: Left ventricular posterior wall; neg T: Negative T; NSTEACS: Non-ST elevation acute coronary syndrome; NSTEMI: Non-ST segment elevation myocardial infarction; ROC: Receiver operator characteristic; UA: Unstable angina}

\section{Acknowledgements}

Not applicable.

\section{Authors' contributions}

This study was conceived and designed by YT, JX and DY. YT and XY were responsible for collection of data or analysis. JX, YT and SYB draft the manuscript. DY checked it and revised critically. All authors read and approved the final manuscript.

\section{Funding}

None.

\section{Availability of data and materials}

All data generated or analysed during this study are included in this published article.

\section{Ethics approval and consent to participate}

The Ethical Committee at The Second affiliated hospital of Dalian Medical University approved this study. The written informed consent was obtained from each participant.

\section{Consent for publication}

Not applicable.

\section{Competing interests}

The authors declare that they have no competing interests.

\section{Author details}

'Department of Cardiology, The Second Affiliated Hospital of Dalian Medical University, Dalian, Liaoning, China. ${ }^{2}$ Department of Cardiology, Shanghai East Hospital, Shanghai Tongji University School of Medicine, Shanghai, China.

Received: 31 March 2020 Accepted: 17 June 2020

Published online: 23 June 2020

\section{References}

1. Paluszkiewicz J, Krasinska B, Milting H, et al. Apical hypertrophic cardiomyopathy: diagnosis, medical and surgical treatment. Kardiochir Torakochirurgia Pol. 2018;15(4):246-53.

2. Jan MF, Todaro MC, Oreto L, et al. Apical hypertrophic cardiomyopathy: present status. Int J Cardiol. 2016;222:745-59.

3. Stephenson E, Monney P, Pugliese F, et al. Ineffective and prolonged apical contraction is associated with chest pain and ischaemia in apical hypertrophic cardiomyopathy. Int J Cardiol. 2018;251:65-70.

4. Malik LH, Singh GD, Amsterdam EA. T-wave tease: apical hypertrophic cardiomyopathy. Am J Med. 2014;127(6):498-500.

5. Olearczyk B, Gollol-Raju N, Menzies DJ. Apical hypertrophic cardiomyopathy mimicking acute coronary syndrome: a case report and review of the literature. Angiology. 2008;59(5):629-31.

6. Lin CS, Chen CH, Ding PY. Apical hypertrophic cardiomyopathy mimicking acute myocardial infarction. Int J Cardiol. 1998;64(3):305-7.
7. Sayin T, Kocum T, Kervancioglu C. Apical hypertrophic cardiomyopathy mimics acute coronary syndrome. Int J Cardiol. 2001;80(1):77-9.

8. Abdin A, Eitel I, de Waha S, et al. Apical hypertrophic cardiomyopathy presenting as acute coronary syndrome. Eur Heart J Acute Cardiovasc Care. 2016;5(3):289-91.

9. Morales J, Giraldo M. Case report: apical variant hypertrophic cardiomyopathy simulating an acute inferior myocardial infarction. J Electrocardiol. 2019;52:35-7.

10. Lakshmanadoss $\mathrm{U}$, Kulkarni A, Balakrishnan S, et al. All that glitters is not gold: apical hypertrophic cardiomyopathy mimicking acute coronary syndrome. Cardiol Res. 2012;3(3):137-9.

11. Rogers PJ, Geib AJ, Cuthbert D, et al. Apical hypertrophic cardiomyopathy: a concerning electrocardiogram in the emergency department. J Emerg Med. 2018;54(6):867-70

12. Chillik I, Ramírez AG, Ordóñez $S$, et al. Electrocardiographic differences between apical hypertrophic cardiomyopathy and apical non-ST segment myocardial infarction. Medicina (B Aires). 2018;78(2):71-5.

13. Parr CJ, Sharma R, Garber PJ. Apical hypertrophic cardiomyopathy treated as ST-elevation myocardial infarction. CJEM. 2018;20(S2):S51-S5.

14. Hughes RK, Knott KD, Malcolmson J, et al. Apical hypertrophic cardiomyopathy: the variant less known. J Am Heart Assoc. 2020;9(5):e015294.

15. Eriksson MJ, Sonnenberg B, Woo A, et al. Long-term outcome in patients with apical hypertrophic cardiomyopathy. J Am Coll Cardiol. 2002;39(4):638-45.

16. Amsterdam EA, Wenger NK, Brindis RG, et al. 2014 AHA/ACC guideline for the Management of Patients with non-ST-elevation acute coronary syndromes: a report of the American College of Cardiology/American Heart Association task force on practice guidelines. J Am Coll Cardiol. 2014;64(24): e139-228.

17. Hannan PJ, Crow RS. Concerning the units for the QT interval corrected by Bazett's formula. Circulation. 1997;96(10):3799.

18. Cokkinos DV, Papantonakos A, Perrakis C, et al. The influence of R-wave amplitude on the degree of ST-segment depression in exercise electrocardiography in the individual patient. Angiology. 1987:38(1):22-7.

19. Khanna S, Sreedharan R, Maheshwari K, et al. Giant T-wave inversions in apical hypertrophic cardiomyopathy. Anesthesiology. 2019;131(2):398-9.

20. Sakamoto T, Tei C, Murayama M, et al. Giant T wave inversion as a manifestation of asymmetrical apical hypertrophy (AAH) of the left ventricle. Echocardiographic and ultrasono-cardiotomographic study. Jpn Heart J. 1976;17(5):611-29.

21. Kitaoka H, Doi Y, Casey SA, et al. Comparison of prevalence of apical hypertrophic cardiomyopathy in Japan and the United States. Am J Cardiol. 2003:92(10):1183-6.

22. Towe EC, Bos JM, Ommen SR, et al. Genotype-phenotype correlations in apical variant hypertrophic cardiomyopathy: genotype-phenotype correlations in apical HCM. Congenit Heart Dis. 2015;10(3):E139-E45.

23. Arad M, Penas-Lado M, Monserrat $L$, et al. Gene mutations in apical hypertrophic cardiomyopathy. Circulation. 2005;112(18):2805-11.

24. Duygu $\mathrm{H}$, Zoghi M, Nalbantgil S, et al. Apical hypertrophic cardiomyopathy might lead to misdiagnosis of ischaemic heart disease. Int I Cardiovasc Imaging. 2008:24(7):675-81.

25. Meghrajani V, Wats K, Saxena A, et al. A 66-year-old female with apical hypertrophic cardiomyopathy presenting with hypertensive crises and type 2 myocardial infarction and a Normal coronary angiogram. Case Rep Cardiol. 2018;2018:7089149.

26. Harari R, Smietana J, Madias JE. Progression of electrocardiographic changes in a patient with apical hypertrophic cardiomyopathy. J Electrocardiol. 2019, 57:132-4

27. Madias JE. Electrocardiogram in apical hypertrophic cardiomyopathy with a speculation as to the mechanism of its features. Neth Heart J. 2013:21 (6): 268-71.

28. Vaidya GN, Antoine S, Imam SH, et al. Reciprocal ST-segment changes in myocardial infarction: ischemia at distance versus Mirror reflection of STelevation. Am J Med Sci. 2018;355(2):162-7.

\section{Publisher's Note}

Springer Nature remains neutral with regard to jurisdictional claims in published maps and institutional affiliations. 